\title{
FERRAMENTA E METODOLOGIA ORIENTADA POR NÍVEIS DE ANÁLISE PARA VISUALIZAÇÃO DE DADOS AMBIENTAIS
}

\author{
L.L. D’Alessandro ${ }^{1,}$; P.L.P Corrêa ${ }^{1}$; L.V. Rizzo ${ }^{2}$; J.L.D Pinaya ${ }^{1}$; M. Oliveira ${ }^{2}$ \\ 1 Departamento de Engenharia de Computação e Sistemas Digitais da Escola Politécnica da \\ Universidade de São Paulo - Universidade de São Paulo \\ Av. Prof. Luciano Gualberto, 158 - São Paulo/SP, CEP.: 05508-010, Brasil \\ Telefone: (11) 3091-0665 - Fax: (11) 3091-5294 \\ 2 Departamento de Ciências Ambientais da Universidade Federal de São Paulo - \\ Universidade Federal de São Paulo \\ Rua Prof. Artur Riedel, 275 - Diadema/SP, CEP.: 09972-270, Brasil \\ Telefone: (11) 3319-3300 - Fax: (11) 3319-3400 \\ *leonardo.dalessandro@usp.br
}

\begin{abstract}
RESUMO: Existe na comunidade de ciências atmosféricas, uma carência pelo desenvolvimento de ferramentas para visualização de dados que integrem diversos conjuntos de informações relacionadas às condições meteorológicas e ambientais. Esse trabalho tem como foco a discussão de técnicas de visualização e gerenciamento de dados ambientais orientadas por uma metodologia de análise ordenada em níveis temporais (níveis de ano, mês e dia). Trata-se de uma sistemática cujo objetivo é fragmentar a análise de dados em parcelas temporais, de forma a evidenciar informações relevantes. Foi desenvolvida uma ferramenta computacional que permitiu a integração dos níveis de análise, facilitando tirada de conclusões. Para a implementação de tal ferramenta, fez-se uso de recursos oferecidos por bibliotecas do Python.
\end{abstract}

PALAVRAS-CHAVE: visualização de dados ambientais; python para ciência de dados; floresta amazônica; GoAmazon; big data.

\begin{abstract}
In the atmospheric science community, there is a need for the development of data visualization tools capable of integrating various sets of information related to meteorological and environmental conditions. This article focuses on the discussion of techniques for visualization and management of environmental data guided by an analysis methodology ordered at temporal levels (year, month and day levels). It is a systematic whose objective is to fragment data analysis into temporary plots, in order to highlight relevant information. To this goal, a computational tool has been developed, which allowed the integration of the analysis levels and facilitated decision-making. For the implementation of such a tool, resources offered by Python libraries have been used.
\end{abstract}

KEYWORDS: visualization of environmental data; python for data science; Amazon rainforest; GoAmazon; big data.

\section{INTRODUÇÃO}

Com a chegada do século XXI, grandes quantidades de informação passaram a ser produzidas diariamente e, com isso, o conceito de big data ganhou relevância. Segundo previsões estatísticas, até 2020, a quantidade de dados gerada será em torno de 44 zettabytes (MARTIN, 2017). 
Nesse cenário, surgiu a necessidade do estudo aprofundado sobre extensos bancos de dados e sobre ciências relacionadas à análise de informações e extração de conhecimentos que auxiliem possíveis tomadas de decisão. Entre elas, destaca-se a data Science (DASGUPTA et al., 2015)

Soma-se a isso a emergência, cada vez maior, de debates sobre questões ambientais e influência humana na natureza. Nesse contexto, tornou-se fundamental a compreensão de causas, influências e consequências dos fenômenos naturais. Ademais, ferramentas tecnológicas se mostraram úteis ao auxiliarem na busca por esses esclarecimentos.

Recentemente, o experimento GoAmazon2014/5, uma colaboração entre o ARM (Atmospheric Radiation Measurement user facility, mantido pelo Departamento de Energia dos EUA - DOE) e várias instituições brasileiras, monitorou as condições atmosféricas em sítios experimentais na Bacia Amazônica, com o objetivo de avaliar o impacto da pluma de poluição do ar de Manaus sobre áreas florestais (MARTIN, 2017).

Como consequência, um conjunto de dados abrangentes e inéditos referentes à região amazônica foi disponibilizado publicamente em bancos de dados mantidos pelo ARM e pelo Laboratório de Física Atmosférica da Universidade de São Paulo (LFA-USP). O projeto foi realizado entre os anos de 2014 e 2015, em que foram realizadas coletas de dados in situ através da instalação de sítios experimentais e coletas por meio de voos sobre a região. Desse modo, entre os dados coletados, há informações das variáveis meteorológicas como temperatura do ar, umidade relativa, velocidade do vento, direção do vento, precipitação e radiação; e de poluentes atmosféricos, como o monóxido de carbono, ozônio, óxidos de nitrogênio, compostos orgânicos voláteis, além de dados de aerossóis e nuvens.

Os dados foram coletados a partir de nove estações de pesquisa, espalhadas pela região da floresta amazônica. As bases experimentais foram posicionadas a fim de se obter dados da pluma urbana de Manaus em diferentes pontos, um destes localizado no centro urbano de Manaus (T1), área em que há uma alta produção e emissão de poluentes (chamados de poluentes primários) pelas indústrias, usinas e automóveis. As estações T2 e T3 são localizadas vento abaixo de Manaus, e sofrem influência da pluma de tal centro urbano, devido à direção preferencial do vento e de queimadas próximas à região. As estações T0t e T0k, por se localizarem próximas entre si, foram englobadas como uma única estação de coleta, denominada $\mathrm{T} 0 \mathrm{z}$, e encontram-se ao norte de Manaus. Enfim, a estação T0a, mais conhecida como torre ATTO, foi posicionada vento acima de Manaus, em uma área com maior preservação da Floresta Amazônica, região denominada background (MARTIN, 2016). As estações estão representadas na Figura 1 (MARTIN, 2017). Os dados meteorológicos utilizados nesse trabalho foram coletados pela estação T0k. 


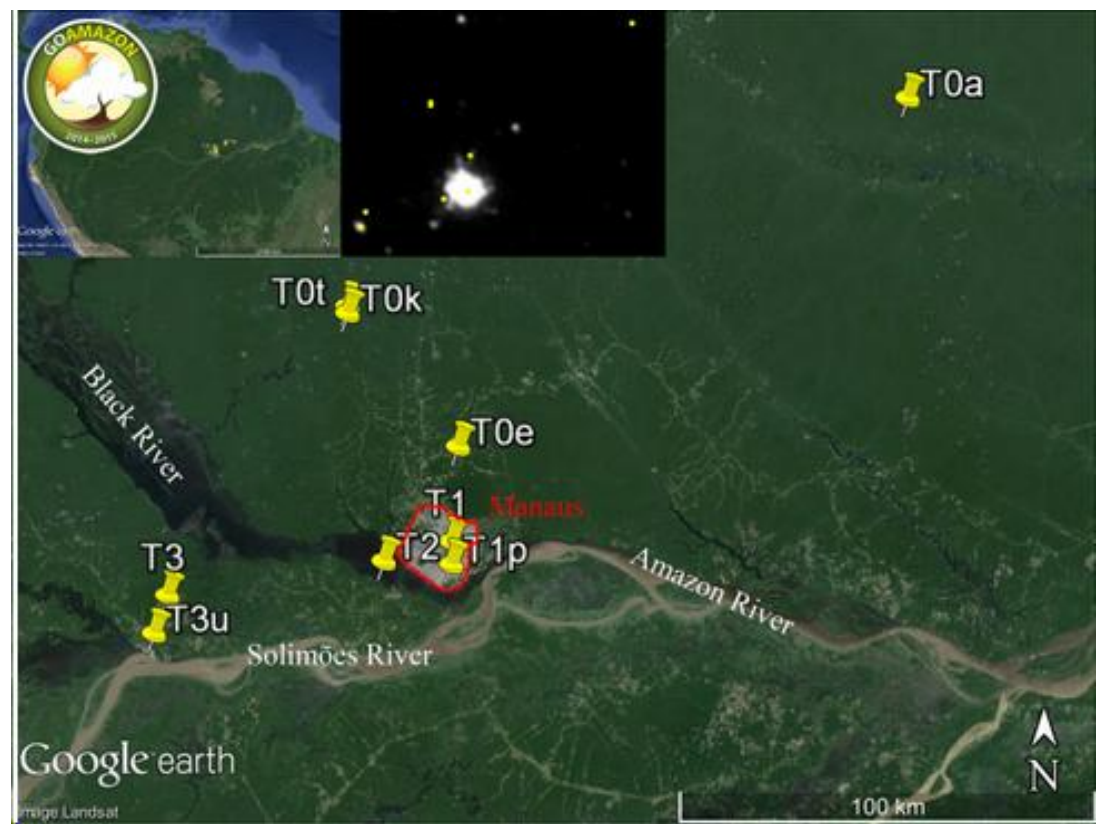

Figura 1. Posição geográfica das estações de coleta.

No âmbito da construção de repositórios especializados, existe, na comunidade de ciências atmosféricas, uma carência pelo desenvolvimento de ferramentas para visualização de dados, integrando diversos conjuntos de informações relacionadas às condições meteorológicas e ambientais citadas anteriormente. A disponibilidade de ferramentas interativas de visualização de dados pode ajudar a desvendar as relações entre variáveis ambientais, auxiliando na produção de conhecimento sobre as complexas interações naturais entre biosfera e atmosfera na Amazônia, bem como avaliar o impacto de atividades antrópicas, como queima de biomassa e poluição urbana.

O presente trabalho traz como foco a discussão sobre técnicas de visualização e gerenciamento de dados ambientais coletados pelo projeto GoAmazon. Na confecção das visualizações de dados foram utilizadas ferramentas computacionais disponibilizadas por bibliotecas do Python, descritas a seguir.

a. Pandas: possibilita a importação de dados em formato $c s v$, o tratamento e organização de dados de data e hora e a criação de dataframes;

b. Seaborn: viabiliza a construção de gráficos de linha, de barra e boxplots de uma ou diversas variáveis;

c. Plotly: semelhantemente ao seaborn, possibilita a construção de gráficos de linha, de barra, e boxplots. Além disso, viabiliza a construção de gráficos de coordenadas paralelas e oferece diversas formas de interatividade com o usuário.

\subsection{Trabalhos Relacionados}

Diversos trabalhos já trataram da análise de temperatura na região da floresta amazônica. Em (GOMES et al., 2015), as visualizações foram feitas por meio de grupos de boxplots, em que cada um dos boxplots se referia a um ano de coleta de dados. Optou-se, em tal trabalho, por utilizar na construção dos gráficos todos os valores de temperatura coletados. Em seguida, os parâmetros foram organizados de acordo com as estações do ano, mesclando-se os dados coletados em todos os anos.

Já no presente trabalho, optou-se pela aplicação da metodologia de cálculo de temperatura diária média proposta pelo INMET (Instituto Nacional de Meteorologia) para a composição dos boxplots em nível anual, com dados mensais organizados. Além disso, foi mais vantajoso, no caso 


\section{ISSN $2447-5378$}

do problema em questão, não mesclar dados entre anos ou estações do ano, já que a proposta é evidenciar detalhes anuais, mensais e diários. Ademais, a utilização da ferramenta de coordenadas paralelas utilizada como forma de aprimorar a visualização de dados atmosféricos, possibilita tanto a análise dos dados de maneira ampla, podendo comparar diferentes variáveis em uma mesma escala de tempo, como também a utilização de estratégias de visualização, como filtros, por exemplo. Os filtros permitem uma análise mais pontual dos dados, em que pode ser útil em análises mais específicas de uma ou duas variáveis.

Por fim, a análise estruturada em níveis proposta pelo presente trabalho e ilustrada adiante facilita a exploração de diversos tipos de variáveis que não somente ambientais. A metodologia e o código desenvolvidos neste trabalho são úteis na análise de diversos tipos de dados, bastando-se realizar adaptações necessárias.

\section{MATERIAIS E MÉTODOS}

\subsection{Arquitetura de Análise}

A análise dos dados proposta neste trabalho foi dividida em níveis: de ano, mês e dia. Inicialmente analisam-se as variáveis em nível de ano, com dados organizados mensalmente. A partir dessa primeira análise define-se um mês de interesse e executa-se a análise no nível de mês, em que os dados são organizados por dia. Mais uma vez, define-se um dia de interesse e, em sua análise os dados são dispostos por hora. Tal estratégia de análise está esquematizada na Figura 2.

Neste trabalho é utilizada a variável de temperatura $\left({ }^{\circ} \mathrm{C}\right)$ a partir dos dados coletados entre 01 de Janeiro de 2014 a 31 de Dezembro de 2014 para os sítios experimentais T0z, T1, T2 e T3. Entretanto, a metodologia proposta pode ser replicada para outras variáveis presentes no banco de dados.

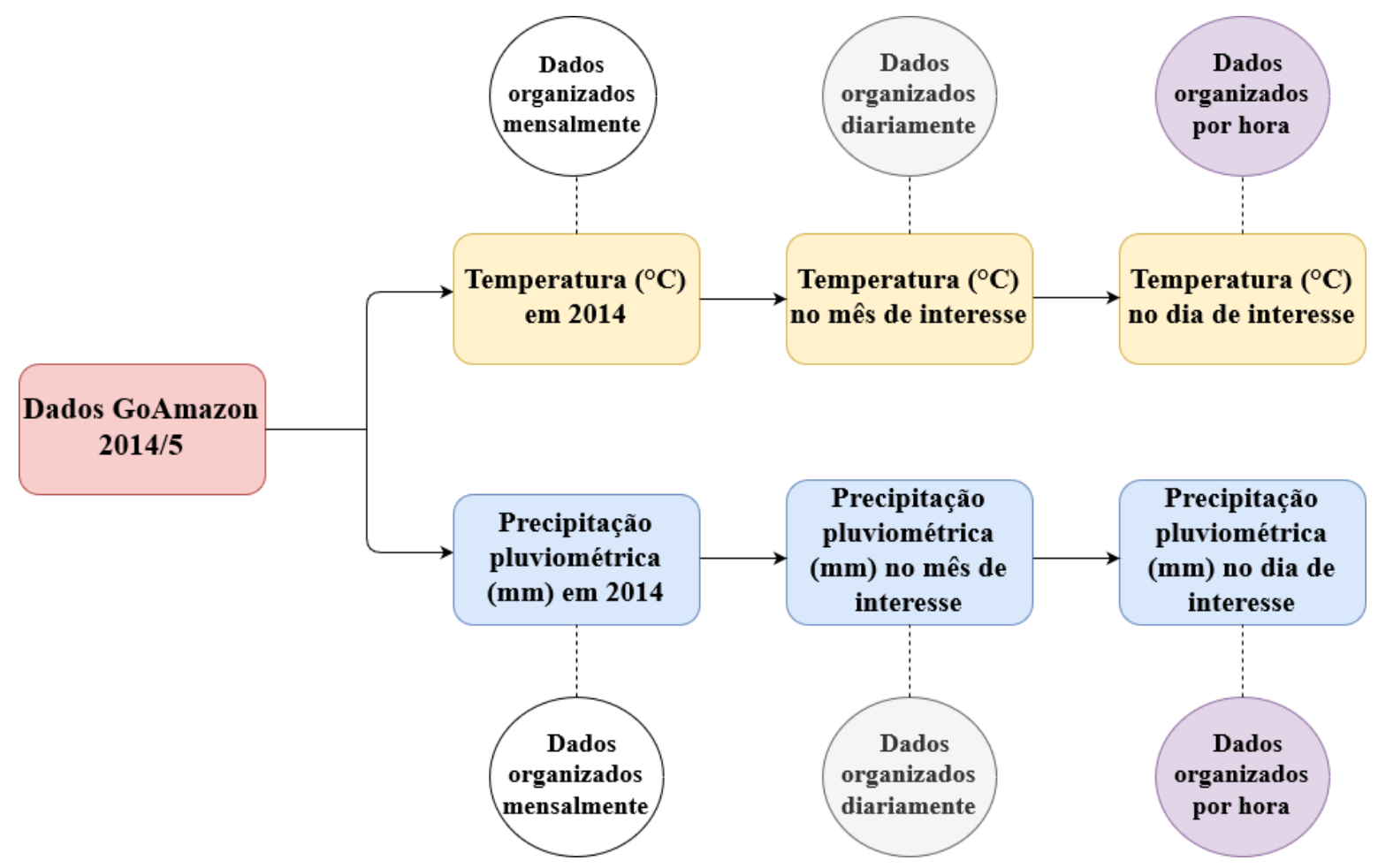

Figura 2. Esquema descritivo de um caso de aplicação da metodologia proposta.

\subsection{Estratégias para Filtragem dos Dados e Construção dos Códigos}


Como mencionado anteriormente, os dados de estudo foram importados, tratados e manipulados por meio da utilização de bibliotecas do Python. Mais especificamente, utilizou-se para tal o Jupyter Notebook, uma aplicação web open-source que permite a criação de diversos códigos na linguagem Python, ordenados em uma lista de células de entradas e saídas.

Inicialmente foi necessário importar as bibliotecas úteis às atividades a serem realizadas. Em seguida, utilizando-se a função pandas.read_csv, oferecida pela biblioteca pandas, importou-se para o Jupyter Notebook os dados em formato .csv armazenados localmente, permitindo assim seu acesso e manipulação.

Já que neste trabalho foram estudadas séries temporais ao longo do ano de 2014, utilizou-se a função pandas.DataFrame.query, cujo objetivo é filtrar os dados conforme o desejado. Além dessa, empregou-se a função pandas.Series.dt.year que permite acessar o ano de uma determinada série de valores de datas. Combinando-se tais funções, foi possível acessar os dados de 2014 e selecioná-los para análise. A seguir, destaca-se uma linha de código que evidencia a aplicação das funções expostas para a filtragem do ano de 2014.

$$
\text { In [1]: DadosDe2014 = DadosGerais.query ('DataDaColeta.dt.year }==2014 ' \text { ') }
$$

Na Figura 3 está exposto um esquema que representa a manipulação geral dos dados, desde sua importação para o Notebook Jupyter até a produção das visualizações desejadas.

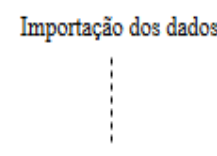

Filtragem dos dados de 2014
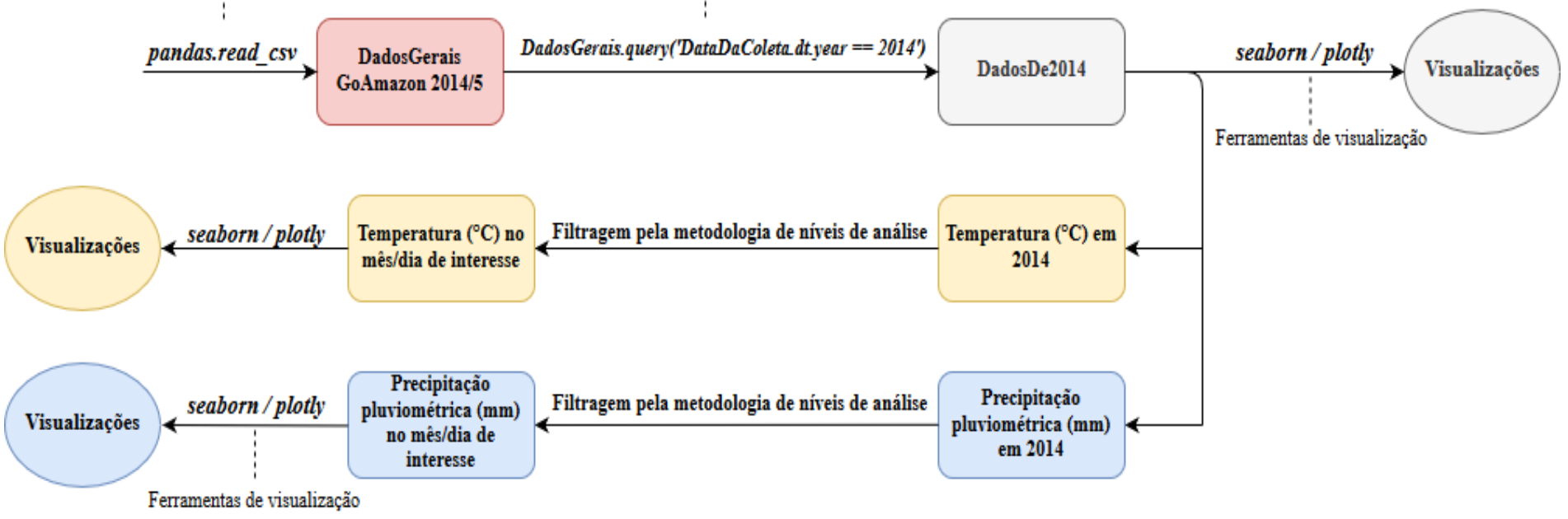

pluviométrica (ma) em 2014

Figura 3. Esquema da manipulação de dados realizada de acordo com a metodologia proposta.

\subsection{Manipulação dos Dados}

Para o cálculo de temperatura diária média utilizou-se o método recomendado pelo Instituto Nacional de Meteorologia (INMET) (MEDEIROS et al., 2013) (Equação 1).

$$
T_{\text {med }}(I N M E T)=\frac{T_{9 h}+T_{\max }+T_{\min }+2 \times T_{21 h}}{5}
$$

Nela,

$T_{\text {med }}$ é a temperatura $\left({ }^{\circ} \mathrm{C}\right)$ média em determinado dia pelo método recomendado pelo INMET;

$T_{9 h}, T_{21 h}$ são temperaturas $\left({ }^{\circ} \mathrm{C}\right)$ observadas respectivamente às $9 \mathrm{~h}$ e $21 \mathrm{~h}$; 
$T_{\max }, T_{\min }$ são temperaturas $\left({ }^{\circ} \mathrm{C}\right)$ máxima e mínima em determinado dia.

Tal método de cálculo da média diária de temperatura foi utilizado para produzir os boxplots para análise do ano de 2014, que serão mostrados adiante.

Ao realizar-se a importação dos dados e a filtragem das variáveis, obteve-se uma tabela em que, em suas colunas as variáveis estão organizadas de acordo com as estações de coleta (T0z, T1, T2 e T3) e nas linhas está exposta cada medição realizada, de acordo com a respectiva data e hora da coleta. Tal organização está representada na Figura 4. Os dados foram coletados e estão dispostos a cada 30 minutos.

Out [9]:

Hora_Local Temp_T0z Temp_T1 Temp_T2 Temp_T3 Rain_T0z Rain_T1 Rain_T2 Rain_T3

\begin{tabular}{|c|c|c|c|c|c|c|c|c|c|}
\hline Datetime & & & & & & & & & \\
\hline 2014-01-01 20:00:00 & 20:00 & 28.77 & 25.47 & $\mathrm{NaN}$ & 26.26 & 0.0 & 0.0 & $\mathrm{NaN}$ & 0.0 \\
\hline 2014-01-01 20:30:00 & $20: 30$ & 28.70 & 25.47 & $\mathrm{NaN}$ & 26.60 & 0.0 & 0.0 & $\mathrm{NaN}$ & 0.0 \\
\hline 2014-01-01 21:00:00 & 21:00 & 28.39 & 25.47 & $\mathrm{NaN}$ & 26.60 & 0.0 & 0.0 & $\mathrm{NaN}$ & 0.0 \\
\hline 2014-01-01 21:30:00 & 21:30 & 27.61 & 25.39 & $\mathrm{NaN}$ & 25.92 & 0.0 & 0.0 & $\mathrm{NaN}$ & 0.0 \\
\hline 2014-01-01 22:00:00 & $22: 00$ & 26.87 & 25.45 & $\mathrm{NaN}$ & 25.57 & 0.0 & 0.0 & $\mathrm{NaN}$ & 0.0 \\
\hline
\end{tabular}

Figura 4. Estrutura de dados resultante após aplicação dos filtros.

Em que:

A coluna "Datetime" exibe a data e hora da coleta;

A coluna "Hora_Local" representa o horário local da coleta;

As colunas "Temp" representam as medidas de temperatura $\left({ }^{\circ} \mathrm{C}\right)$ em suas respectivas estações de coleta (T0z, T1, T2 e T3);

As colunas "Rain" representam as medidas de precipitação pluviométrica $(\mathrm{mm})$ em suas respectivas estações de coleta (T0z, T1, T2 e T3), que não serão utilizadas no presente trabalho.

Assim, para retratar a metodologia proposta por este trabalho e a ferramenta de análise construída foram feitas visualizações de dados referentes à estação T0k, que compõe a estação T0z e localiza-se ao norte de Manaus.

\subsection{Níveis de Análise}

Como mencionado anteriormente, a análise foi dividida em níveis: de ano, em que as variáveis são exploradas mensalmente; de mês, em que são analisadas diariamente; e de dia, em que os parâmetros são estudados por hora. Mais especificamente, no caso de análise de temperatura nos níveis de ano e de mês, foram construídos boxplots. No caso da análise no nível de ano, os boxplots são constituídos por valores de médias diárias calculadas pelo método do INMET. Já para o nível de mês, os boxplots são formados por todas as medidas de temperatura coletadas em cada dia. Enfim, na análise no nível de dia foi criado um gráfico de linha que exibe todos os valores de temperatura coletados em cada hora.

\subsection{Ferramenta de Coordenadas Paralelas}

Além de boxplots, gráficos de barras e gráficos de linha, tipos já difundidos na visualização de dados, foram produzidos gráficos do tipo coordenadas paralelas. Nesse tipo de representação, cada eixo paralelo indica uma variável a ser estudada. As instâncias de dados são polilinhas que interceptam os eixos em uma posição determinada pelo valor da variável associada ao eixo 
(INSLBERG, 1985). A seguir, destacam-se algumas linhas de código responsáveis pela definição dos dados a serem exibidos no gráfico de coordenadas paralelas.

In $[18]:$ data $=[$

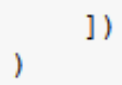

Nas instruções, é possível notar a determinação das variáveis a serem exibidas, como Temp_TOz, que representa a temperatura na estação de coleta $\mathrm{TOz}$, além da variável Data.1, que quando combinada com os comandos .dt.month e .dt.day (oferecidos pela biblioteca Pandas) indica respectivamente o mês e o dia em que certo dado foi coletado. O resultado será exibido adiante, na Figura 9.

\section{RESULTADOS E DISCUSSÃO}

\subsection{Resultados para Temperatura em T0z}

Primeiramente, a partir da biblioteca Plotly foram construídos boxplots ordenados em meses, representando a análise no nível de ano. O resultado está exibido na Figura 5. Com essa primeira análise, é possível definir que fevereiro foi o mês mais frio de 2014 e que setembro foi o mês mais quente na estação T0z.

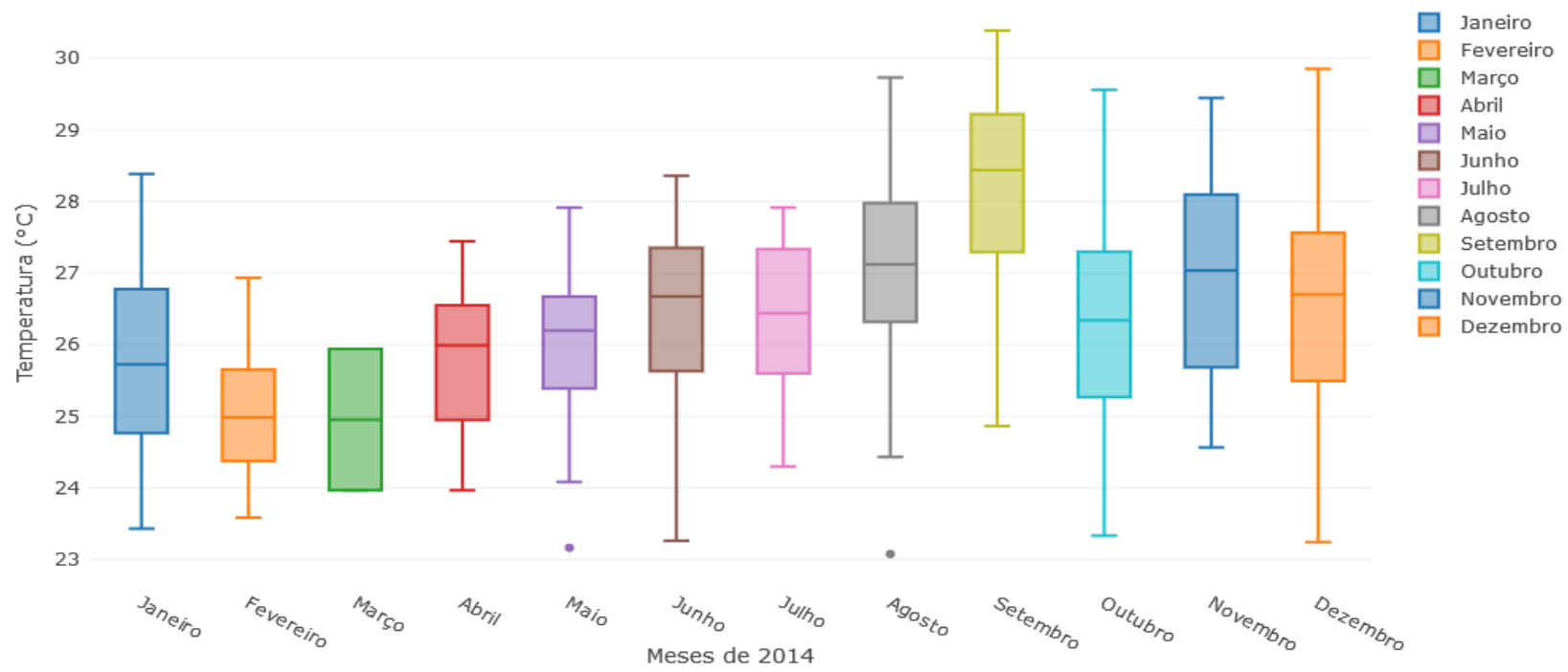

Figura 5. Boxplots de temperatura diária média (INMET) na estação T0z em 2014.

Com essas informações, é possível passar à análise para a escala temporal mensal. As Figuras 6 e 7 ilustram respectivamente a temperatura na estação T0z durante os meses de fevereiro e 


\section{ISSN $2447-5378$}

setembro de 2014. A biblioteca utilizada na confecção de tais gráficos foi o seaborn. Na confecção de tais boxplots utilizou-se todos os dados de temperatura coletados na estação T0z no ano de 2014.

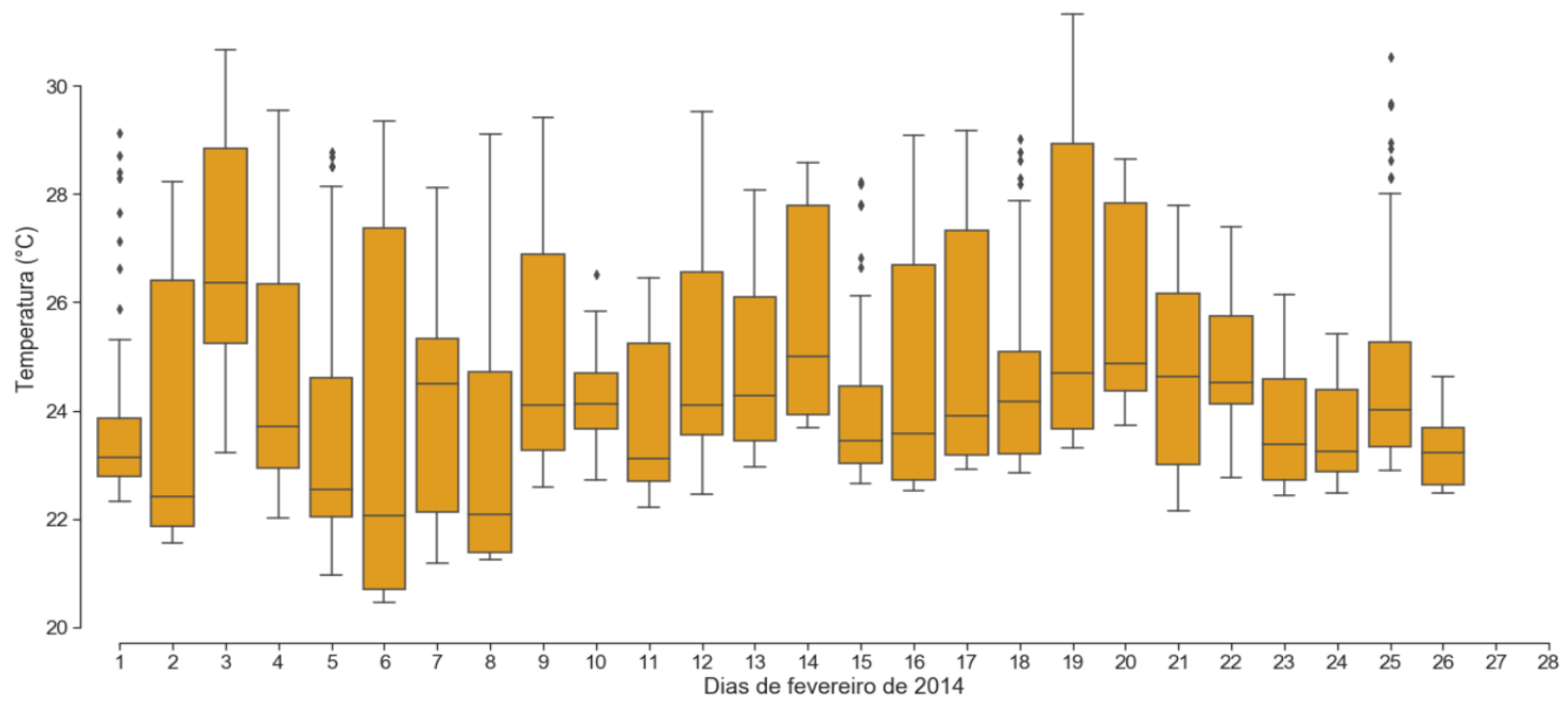

Figura 6. Boxplots da temperatura na estação de coleta T0z em fevereiro de 2014.
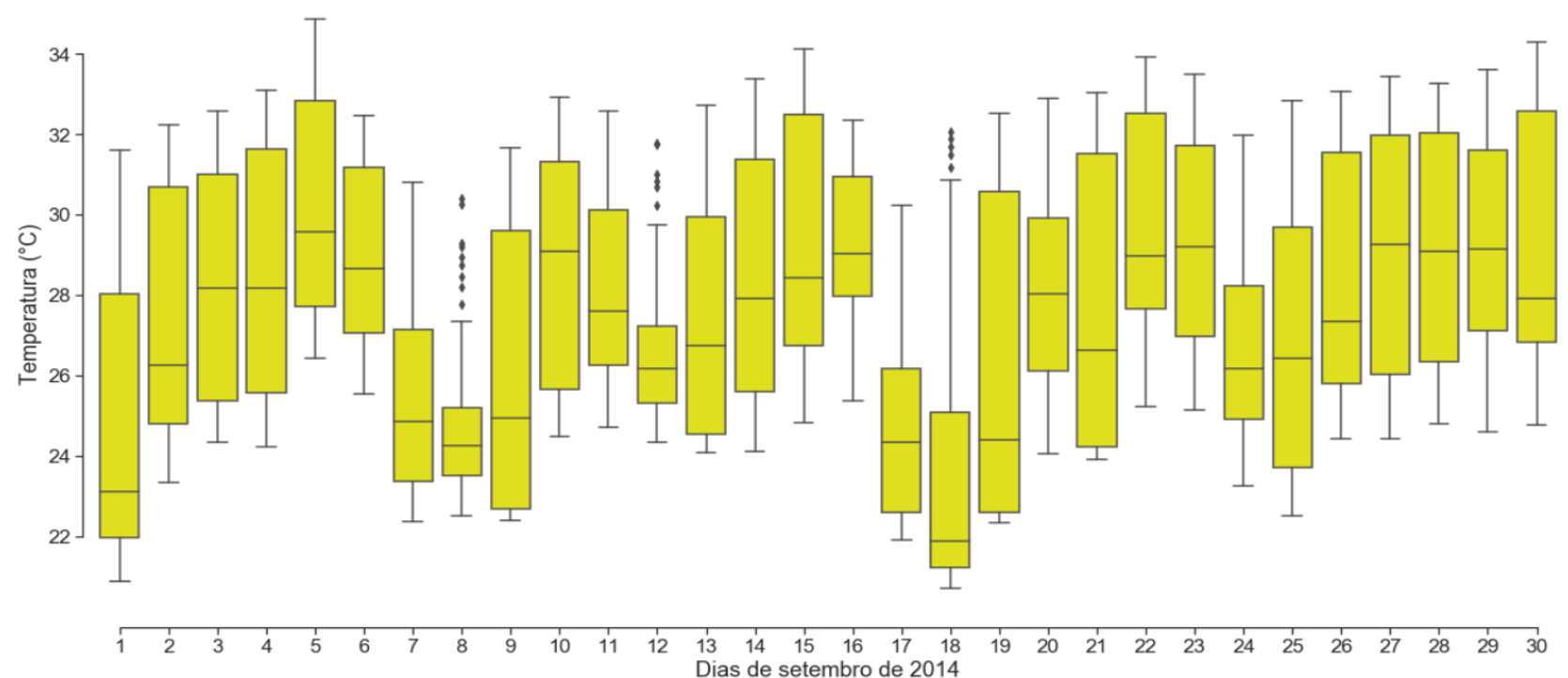

Figura 7. Boxplots da temperatura na estação de coleta T0z em setembro de 2014.

A análise em diferentes escalas de tempo, diárias ou mensais, permite identificar a ocorrência de fenômenos climáticos e meteorológicos de naturezas distintas e seu comportamento em duas estações do ano bem definidas na região. As temperaturas para o mês de fevereiro, por exemplo, representadas no gráfico da Figura 6, permitem analisar a estação chuvosa em Manaus, com mais ocorrência de nebulosidade e temperaturas mais baixas do longo do mês (MARTIN, 2017), sendo o dia 06 de fevereiro de 2014 o de menor temperatura.

Entretanto, em setembro (Figura 7), as temperaturas médias são mais altas se comparadas com o primeiro período. $\mathrm{O}$ mês de setembro está compreendido em um período que corresponde à estação seca do ano, quando há menor ocorrência de precipitação na região. Os dados podem ser analisados em diferentes escalas temporais. Os dias mais frios de setembro, por exemplo, podem estar relacionados à ocorrência de fenômenos meteorológicos de mesoescala (que, em geral, duram poucos dias), como entre os dias 7 a 8 e 17 a 18 de setembro. 
Além das escalas temporais, as variações nas condições atmosféricas também podem ocorrer devido a diferentes fenômenos meteorológicos. Algumas variações dependem somente da latitude do local: como por exemplo, as variações sazonais (dentro de um ano, relacionadas às estações do ano) e variações diárias (dentro de um dia, relacionadas principalmente ao ciclo diurno da radiação solar). Outras variações são consequência da ação de fenômenos meteorológicos sinópticos, que são sistemas que possuem centenas de quilômetros de extensão, e que persistem por até cerca de 15 dias na região, podendo modificar as condições mais comuns em determinado período, e que podem ser de mesoescala (regionais, e que persistem por poucos dias) ou de microescala (locais, e que duram apenas algumas horas).

Passando à análise em nível de dia, obtêm-se os resultados exibidos na Figura 8, referente ao dia mais quente de setembro de 2014 na estação T0z. A biblioteca utilizada na confecção de tal gráfico foi o Plotly. A variação da temperatura ao longo do dia ocorre devido a presença de maior radiação solar e o período de exposição, considerando uma região com alta densidade de floresta e baixa presença antrópica. A biblioteca utilizada na confecção de tal gráfico foi o Plotly.

O pico noturno de temperatura observado em 05 de setembro (Figura 8) pode estar relacionado à ocorrência de um fenômeno de microescala ou de mesoescala.

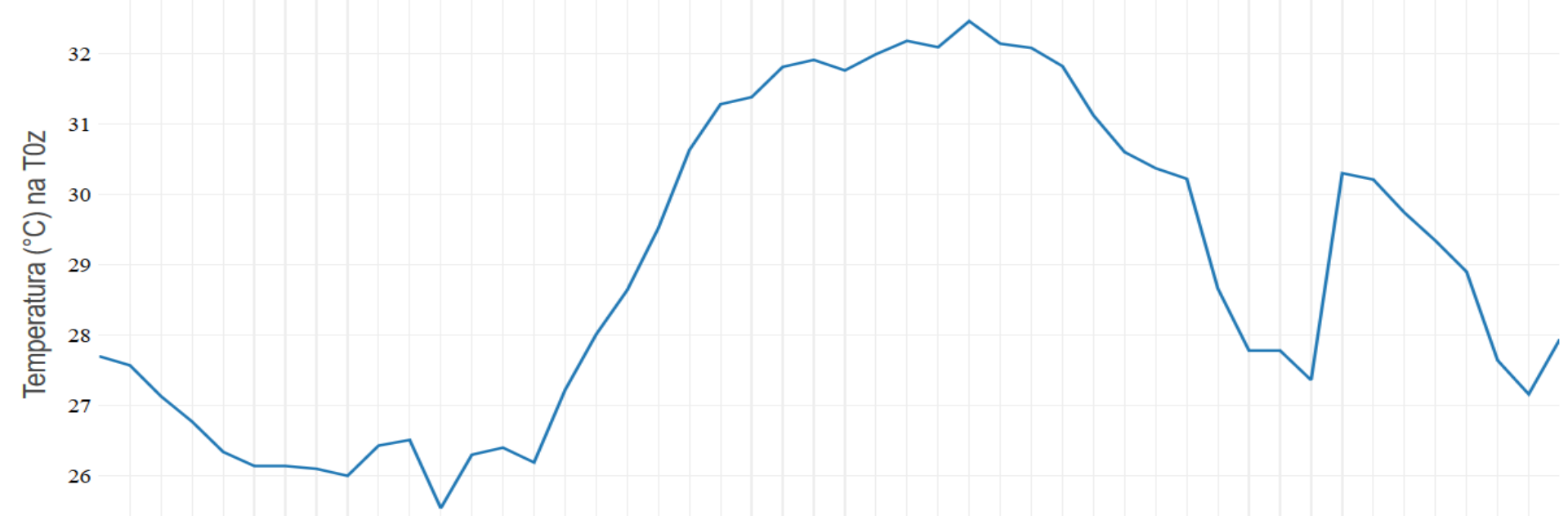

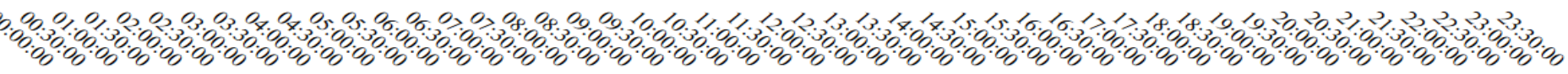
Hora Local

Figura 8. Temperatura na estação de coleta T0z no dia 05/09/2014.

\subsection{Resultados Obtidos por meio da Ferramenta de Coordenadas Paralelas, Disponibilizada pela Biblioteca Plotly}

A fim de ilustrar a utilidade dos gráficos de coordenadas paralelas segundo a metodologia de análise proposta no presente trabalho, foram usadas como exemplo as temperaturas médias diárias em 2014, calculadas pelo método recomendado pelo INMET na estação T0z. Sendo assim, construiu-se um gráfico geral, em que são exibidas as variáveis de temperatura $\left({ }^{\circ} \mathrm{C}\right)$, os meses do ano (representados de 1 a 12) e os dias (representados de 1 a 31). 


\section{ISSN $2447-5378$}

Temperatura média diária de TOZ $\left({ }^{\circ} \mathrm{C}\right)$ pelo método da INMET

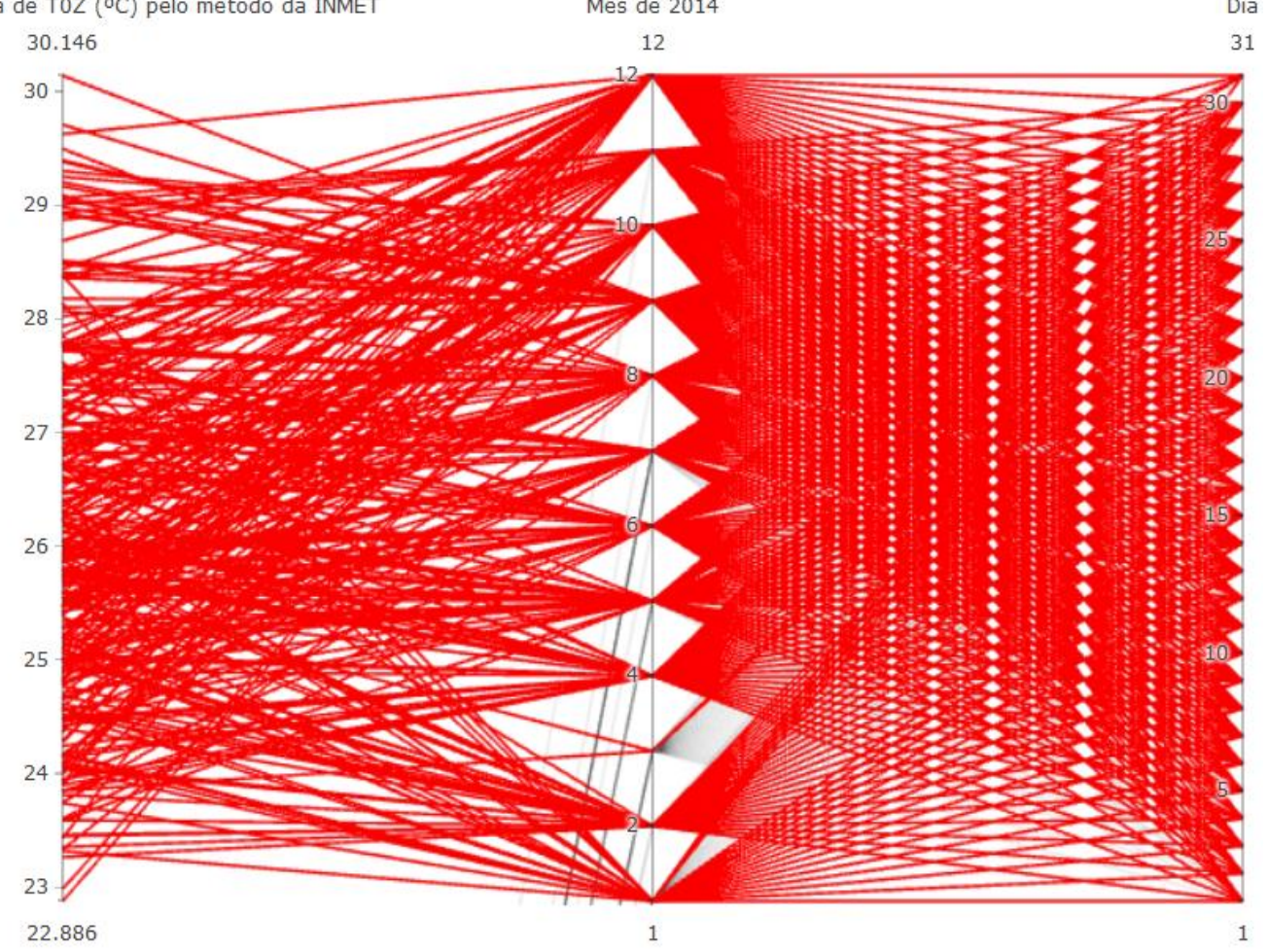

Figura 9. Gráfico de coordenadas paralelas de 2014 sem aplicação de filtros.

Em seguida, aplicou-se uma funcionalidade de filtro interativo disponibilizada pela biblioteca Plotly. Com ela foi possível selecionar e analisar as temperaturas diárias médias mais altas, aquelas entre $30,2^{\circ} \mathrm{C}$ e $28^{\circ} \mathrm{C}$. O filtro é representado pela cor rosa na Figura 10. As linhas que permanecem na cor vermelha após a aplicação do filtro são aquelas que correspondem à faixa de temperaturas selecionadas. Ao aplicar-se tal filtro é possível notar que, de fato, setembro foi o mês mais quente de 2014 na estação de coleta em questão. Isso é verificado por meio da grande concentração de linhas vermelhas em tal mês ao selecionarem-se as temperaturas maiores. 


\section{ISSN $2447-5378$}

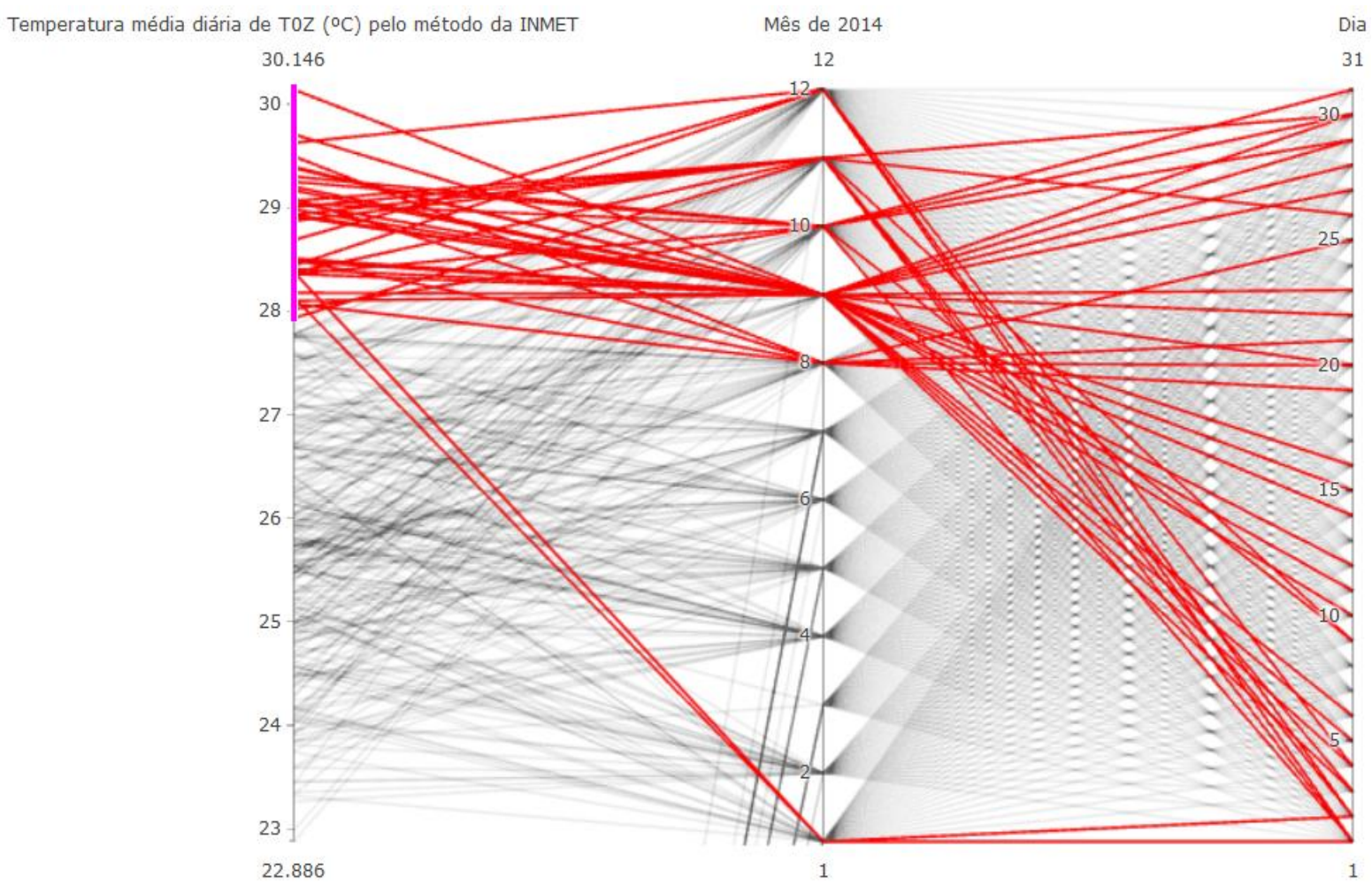

Figura 10. Gráfico de coordenadas paralelas de 2014 com filtro de temperaturas elevadas.

Em seguida, aplicou-se o filtro para temperaturas menores (entre $23,9^{\circ} \mathrm{C}$ e $22,9^{\circ} \mathrm{C}$ ) e observou-se que, de fato, fevereiro foi o mês mais frio de 2014, devido à alta concentração de linhas de cor vermelha em tal mês. O resultado é exibido na Figura 11.

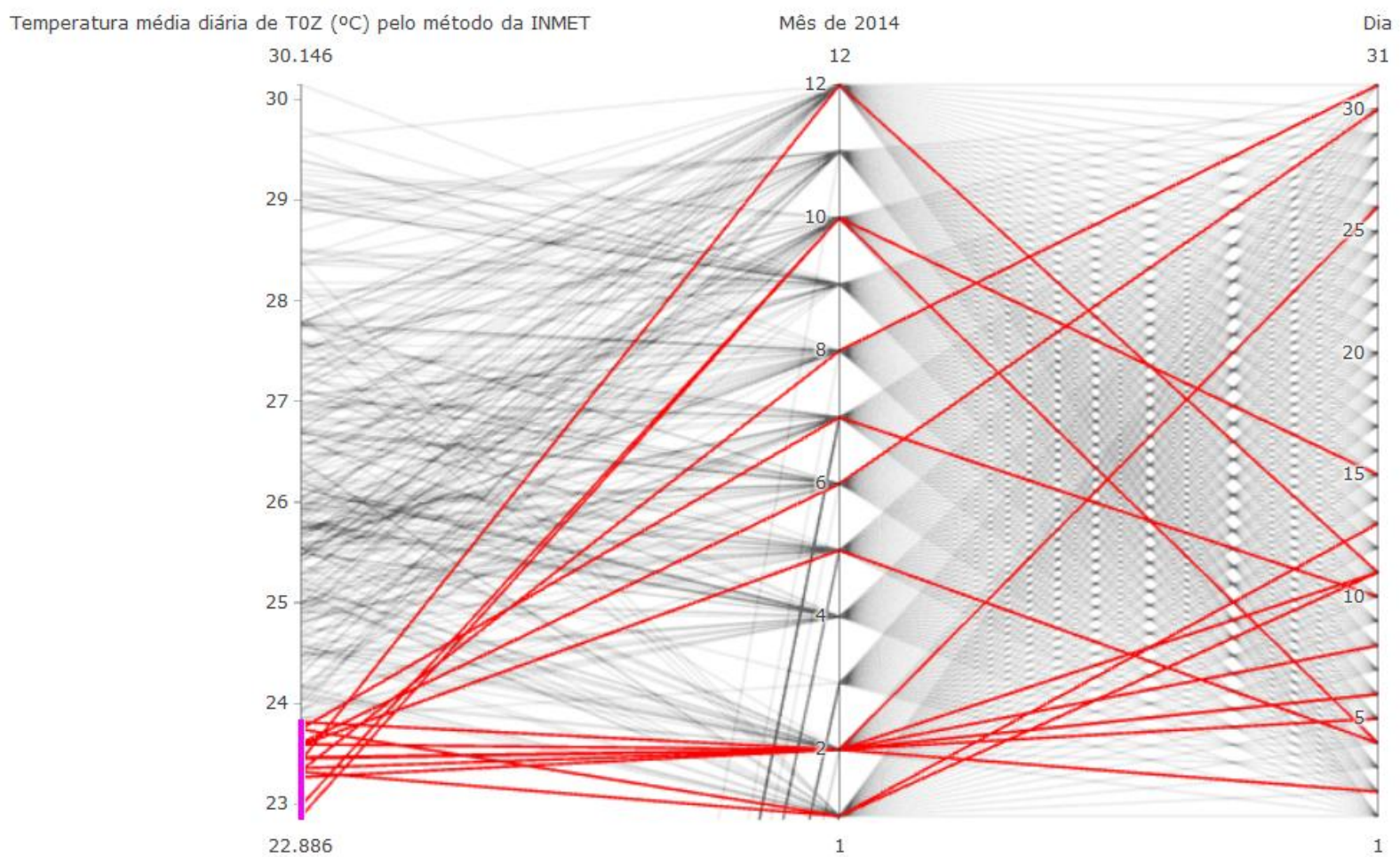

Figura 11. Gráfico de coordenadas paralelas de 2014 com filtro de menores temperaturas. 


\subsection{Vantagens da Utilização do Jupyter Notebook}

As vantagens na utilização do software Jupyter Notebook, assim como da linguagem de programação Python, foram evidenciadas ao longo deste trabalho. Primeiramente, o Jupyter Notebook se trata de uma ferramenta open source e gratuita. Tal característica permite a universalização dos códigos, uma vez que estes gerarão os mesmos resultados em qualquer dispositivo que execute o programa. Além disso, as bibliotecas do Python oferecem uma vasta gama de opções de gráficos e personalizações, que foram úteis na confecção dos boxplots e gráficos de coordenadas paralelas presentes neste trabalho.

\section{CONCLUSÃO}

Neste trabalho avaliou-se o uso de uma metodologia de análise hierárquica ordenada em níveis de ano, de mês e de dia que tem como objetivo direcionar a análise de variáveis em pontos de interesse. Tal tipo de abordagem permite uma rápida identificação de eventos relevantes. $\mathrm{O}$ código desenvolvido no presente trabalho pode auxiliar na análise de outros tipos de variáveis, desde que essas tenham o tempo como parâmetro de referência. No caso do estudo de temperaturas, destaca-se a importância de adotarem-se critérios padronizados de manipulação de dados, como por exemplo, o método de cálculo de temperatura diária média sugerido pelo INMET. Isso permite análises comparativas com outros dados e estudos difundidos na literatura científica.

\section{AGRADECIMENTOS}

Esta pesquisa é apoiada pela Fundação de Amparo à Pesquisa do Estado de São Paulo (FAPESP) Programa de Pesquisa em eScience (processo: 2016/04982-0). Este trabalho foi realizado com o apoio do Itaú Unibanco S.A., por meio do Programa de Bolsas Itaú (PBI), vinculado ao Centro de Ciência de Dados da Escola Politécnica da USP.

\section{REFERÊNCIAS BIBLIOGRÁFICAS}

DASGUPTA, A.; POCO, J.; WEI, Y.; COOK, R.; BERITINI, E.; SILVA, C. Bridging theory with practice: An exploratory study of visualization use and design for climate model comparison. IEEE Transactions on Visualization and Computer Graphics, 2015. v. 21, n. 9, p. 996-1014.

GOMES, A.; COSTA, M.; COUTINHO, M.; VALE, R.; SANTOS, M.; SILVA, J.; FITZJARRALD, D. Análise estatística das tendências de elevação nas séries de temperatura média máxima na Amazônia central: estudo de caso para a região do oeste do Pará. Revista Brasileira de Climatologia, 2015.

INSLBERG, A. The plane with parallel coordinates. The Visual Computer, 1985. p. 69-70.

MARTIN, S. The Green Ocean Amazon Experiment (GoAmazon2014/5) Observes Pollution Affecting Gases, Aerosols, Clouds, and Rainfall over the RainForest. American Meteorological Society, 2017.

MARTIN, S. Introduction: Observations and Modeling of the Green Ocean Amazon (GoAmazon2014/5). Copernicus Publications. Atmospheric Chemistry and Physics, 2016.

MEDEIROS, R.; LIMA, T.; KASSAR, C.; SILVA, V. Metodologias de cálculo da temperatura média diária do ar: aplicação para os municípios de Parnaíba, Picos e Gilbués, PI. Revista Brasileira de Agricultura Irrigada, 2017. v. 6, n. 4, p. 283-295. 